\title{
Wikileaks: costruire la trasparenza - Analisi del video "Collateral Murder"
}

\author{
Isabella Pezzini
}

Riassunto: L'articolo esamina la strategia comunicativa adottata da Wikileaks in occasione della diffusione del video "Collateral murder" (3 aprile 2010), in cui denunciava il comportamento dell'aviazione americana nella guerra in Iraq e si proponeva come un nuovo soggetto della controcomunicazione globale. Analizzando i modi in cui il video fu costruito a partire dalle registrazioni delle azioni militari sottratte agli stessi USA, emerge il rovesciamento narrativo che Wikileaks riuscì a ottenere, presentando la propria struttura come il vero Destinante custode dei valori condivisi dall'opinione pubblica mondiale, smascherando viceversa gli USA come Antidestinante.

Parole chiave: Wikileaks; transparenza comunicativa; strategia di veridizione; argomentazione audiovisiva.

Abstract: WikiLeaks: building transparency - Analysis of the video "Collateral Murder" - This paper examines the communication strategy adopted by WikiLeaks at the time of the dissemination of the video "Collateral Murder" (3 April 2010), which denounces the behavior of the American air force during the war in Iraq and presents itself as a new instrument for global counterinformation. Analyzing the ways in which the video was created based on records of military operations stolen from the USA itself, this article describes the counternarrative discovered by WikiLeaks, which presents its own structure as the true destinator of the values shared by global public opinion, while unmasking the USA as the anti-destinator.

Keywords: WikiLeaks; communication transparency; veridiction strategy; audiovisual reasoning.

Il flop dell'atteso (e per la verità deludente) film hollywoodiano su Julian Assange e gli exploit mediatici di Wikileaks - The Fifth Estate di Bill Condon (USA, 2013) - è stato letto dalla stampa come I'ultimo indizio di una generale caduta di interesse da parte dell'opinione pubblica nei confronti di una vicenda che ha comunque segnato in modo irreversibile la storia del giornalismo e dell'informazione. ${ }^{1}$ Al tempo stesso, le critiche

1 Così una parte della definzione che si trova in Wikipedia, interessante per la sua popolarità in rete: "WikiLeaks (dall'inglese "leak", "perdita", "fuga [di notizie]") è un'organizzazione internazionale senza scopo di lucro che riceve in modo anonimo, grazie a un contenitore protetto da un potente sistema di cifratura, documenti 
al film rappresentano un'ulteriore testimonianza della controversia suscitata dalla figura di Julian Assange, un corpus sempre più vasto, che comprende un numero impressionante di libri, film, persino graphic novel e interventi di varia altra natura, in conflitto fra loro per tesi e interpretazioni. II mancato successo del film segue quello dell'autobiografia dello stesso Assange, la sua sconfitta politica alle elezioni australiane e infine la condanna a 35 anni di reclusione di Bradley Manney, uno dei suoi principali informatori. A quest'ultimo non è stato infatti riconosciuto lo statuto onorevole di whistleblower, e cioè di "colui che denuncia un fatto grave" per il bene comune, bensì sostanzialmente quello di traditore della patria. ${ }^{2}$

Bradley Manney, analista dell'intelligence americana, aveva passato a Wikileaks, dopo averli scaricati dai computer su un CD apparentemente di Lady Gaga, circa 700.000 files considerati "fired", cioè secretati dall'amministrazione americana. Fra questi si trovava il celeberrimo filmato degli elicotteri Apaches in azione a Bagdad in una brutta giornata del 2007, il 12 luglio, su cui ci soffermeremo qui. La video registrazione documenta come soldati americani, scambiando cineprese per bazooka, abbiano sparato a un gruppo di civili che si aggirava per le vie della città, uccidendone deliberatamente ben 12 . Fra di loro c'erano anche due giornalisti dell'agenzia di stampa Reuter: non contenti della prima azione, gli stessi soldati fecero altri morti e feriti sparando anche ai soccorritori, fermandosi solo una volta accortisi che fra i feriti c'erano dei bambini.

Dalla registrazione USA di 38 minuti Assange e i suoi compagni ricavarono un video, intitolato appunto Collateral murder, di 17 minuti, che venne proiettato durante una conferenza stampa il 5 aprile 2010 suscitando enorme scalpore (risulta postato su Youtube il 3 aprile). Ritorcendo la documentazione contro i suoi stessi autori, ne usciva una precisa denuncia della condotta USA nella guerra irakena, del cinismo delle sue

coperti da segreto (segreto di stato, segreto militare, segreto industriale, segreto bancario) e poi li carica sul proprio sito web. WikiLeaks riceve, in genere, documenti di carattere governativo o aziendale da fonti coperte dall'anonimato. Il sito è curato da giornalisti, attivisti, scienziati. Comunque i cittadini di ogni parte del mondo possono inviare (sono anzi invitati a farlo) materiale 'che porti alla luce comportamenti non etici di governi e aziende' tenuti nascosti. Gran parte dello staff del sito, come gli stessi fondatori del progetto, rimane anonima. L'obiettivo dell'organizzazione è di assicurare che gli informatori NON vengano perseguiti per la diffusione di documenti sensibili. L'organizzazione dichiara di verificare l'autenticità del materiale prima di pubblicarlo e di preservare l'anonimato degli informatori e di tutti coloro che sono implicati nella 'fuga di notizie'. Nonostante il prefisso, il progetto non è un wiki e non ha alcun legame con Wikimedia Foundation, l'organizzazione senza fini di lucro che possiede i server di Wikipedia. WikiLeaks, per scelta, non ha alcuna sede ufficiale. Infatti vuole essere «una versione irrintracciabile di Wikipedia che consenta la pubblicazione e l'analisi di massa di documentazione riservata»". Lo scopo ultimo è quello della trasparenza da parte dei governi quale garanzia di giustizia, di etica e di una più forte democrazia.

2 Con il termine di whisteblower - tradotto in italiano come "gola profonda" - si definisce chi denuncia pubblicamente e riferisce alle autorità attività illecite o fraudolente all'interno del governo, di un'organizzazione pubblica o privata o di un'azienda, nel quadro di una generale ideologia della necessità di una collaborazione individuale/collettiva al mantenimento dell'ordine sociale. Le rivelazioni possono essere di varia natura: violazione di una legge o di un regolamento, minaccia $d^{\prime}$ interesse pubblico come in caso di corruzione o frode. Si possono denunciare tali condotte all'interno dell'organizzazione stessa, o renderle pubbliche attraverso i media o le associazioni che si occupano dei problemi in questione. Spesso le gole profonde si espongono a ritorsioni e rivalse da parte dell'istituzione che hanno accusato o di altre organizzazioni correlate (vedi il caso dei pentiti, o in certi casi anche dei semplici testimoni, in Italia, che per legge dovrebbero beneficiare di uno specifico programma di protezione). 
truppe e degli atti criminali che esse potevano avere compiuto "di routine". Al tempo stesso era un saggio e una prova dello scottante interesse dell'enorme materiale di cui era entrato in possesso Wikileaks, che come ora sappiamo sarebbe stato caricato in rete senza tutte le verifiche promesse. La giustificazione fornita dall'affermazione di principio: "Wikileaks non interviene sui materiali che riceve", nel caso specifico fu preventivamente contraddetta dall'elaborazione del video, che si rivelò del resto una mossa molto efficace dal punto di vista comunicativo. Per Wikileaks fu il momento di massima fama e reputazione, in cui realizzò un notevole salto di qualità grazie all'alleanza con i grandi e autorevoli giornali della stampa internazionale, dal New York Times a Die Spiegel, a The Guardian, The Economist etc. ${ }^{3}$

Wikileaks in effetti si trasforma, modifica il proprio statuto di soggetto semiotico, proprio nel percorso di trattamento di questo video, ben al di là della decrittazione iniziale del filmato USA. E' quanto cercheremo di ricostruire a partire dalle tracce iscritte nel testo finale. Come vedremo, Wikileaks opera secondo una strategia di manipolazione molto classica, identificabile sostanzialmente nell'uso coordinato di alcune modalità discorsive o figure ricorrenti: la denuncia; il racconto; la prova; la testimonianza. Ne deriva, fra I'altro, una radicale trasformazione nel modo di pensare, enunciare e soprattutto praticare la cosiddetta trasparenza comunicativa: se inizialmente Wikileaks aveva manifestato l'idea che essa coincidesse con la pubblicazione "tale e quale" dei documenti ricevuti, che smascheravano chi li manteneva segreti, nelle scelte che in seguito ha operato dimostra di avere maturato la ben chiara consapevolezza che la trasparenza è semmai il risultato di una precisa strategia discorsiva, e non un astratto apriori. E che, come sottolinea la riflessione semiotica, porta necessariamente con sé un certo grado di corrispettiva opacità, trattandosi in ogni caso di effetti in una certa misura correlati (LOZANO, 2012).

Iniziamo dalla figura della denuncia, che ingloba parzialmente anche le altre. Dalla registrazione originale di 38 minuti Wikileaks ricava un video di 17 sulla base di una serie di operazioni di editing, e cioè in termini semiotici di testualizzazione e ri-enunciazione, orientate a uno spettatore-modello, che dovrà essere non solo edotto dei fatti denunciati, ma anche indignarsi e reagire contro gli autori di questi ultimi. Possiamo ripercorrere brevemente queste diverse operazioni, che hanno tutte a che fare con l'istituzione di un testo, le cui caratteristiche strutturali di coerenza formale o di coesione interna, di tenuta (MARRONE, 2011), dipendono anzitutto dal dispositivo di chiusura che lo regola. Vediamo anzitutto I'inizio e la fine, I'istituzione di una soglia o bordo testuale che ha una doppia funzione, verso l'interno, di contenimento, e verso l'esterno, di orientamento enunciazionale dello spettatore (GENETTE, 1987).

TITOLAZIONE/COPYRIGHT/ESERGO: I titoli di testa - Collateral murder I'apposizione di un copyright (SUNSHINE, 2010) e una citazione di Orwell in esergo sono marche di autorialità e di proprietà intellettuale. Istituiscono un asse di enunciazione

3 Ringrazio Giuseppe Anzera ( Dipartimento CORIS, Sapienza Università di Roma) per le riflessioni e i materiali che mi ha messo a disposizione. 
in cui l'enunciatore si qualifica e offre un frame all'enunciatario, una chiave interpretativa globale a quanto sta per vedere, un patto di visione/lettura specifico. La citazione recita: "Political language is designed to make lies sound truthful and murder respectable, and to give the appearance of solidity to pure wind": I'isotopia proposta come cornice generale è dunque quella della menzogna e della veridizione, dello scollamento tra essere e apparire che caratterizza in generale il discorso politico, e che avrà qui un'applicazione particolare nel caso degli USA.

COSTRUZIONE del PROLOGO e dell'EPILOGO. Iniziamo dal primo, della durata di 2 minuti e 45: una serie di cartelli fissi (che permangono sullo schermo abbastanza a lungo per poter essere letti) procede a un inquadramento spazio-temporale e alla presentazione dei principali attori della vicenda. Questo avviene in particolare attraverso I'IDENTIFICAZIONE delle VITTIME, e il conseguente SMASCHERAMENTO dei PERSECUTORI.

Nel primo cartello la notizia: il 12 luglio 2007 a Bagdad una dozzina di civili, identificati come "anti-Iraqi forces" o "insurgents" malgrado l'attitudine tranquilla, furono uccisi e due bambini feriti da soldati USA a bordo di due elicotteri Apaches. Due delle vittime, per lo più rimaste sconosciute, erano in realtà dipendenti dall'agenzia di stampa Reuters, e cioè Saeed Chmaagh e Namir Noor-Elden. Il cartello seguente reca una fotografia del figlio di Saeed e una didascalia di commento. II ragazzo piangente stringe al petto una grande foto del padre: la didascalia ci informa che quest'ultimo era un autista-assistente della Reuters, aveva 40 anni, ha lasciato la moglie e quattro figli. Segue un ritratto di Namir, sorridente, in posa sul tetto di una camionetta con la macchina fotografica in mano: aveva 22 anni, veniva da una famiglia di giornalisti e era uno dei più stimati fotografi dell'Iraq (Fig.1).

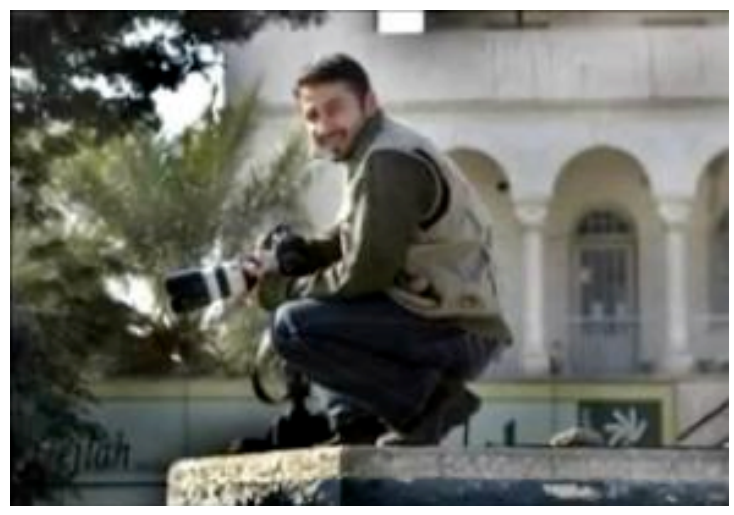

Fig.1. La fotografia di Namir, fotografo ucciso nell'azione

Nel cartello seguente un'altra immagine ritrae Namir insieme a Steve Crisp, suo caporedattore, che lo vide uscire con Saeed per la sua ultima missione. Infine si dice della domanda della Reuters di aprire un'inchiesta e della risposta USA secondo 
la quale I'azione era conforme alle regole d'ingaggio dei soldati. La Reuters allora aveva chiesto senza successo al Freedom Information Act di poter avere la registrazione filmata dell'azione. Ma ecco, e siamo all'ultimo cartello, che "Wikileaks exposes Collater Murder": sulla sinistra accanto al testo in versione macro c'è il logo di Wikileaks, la clessidra azzurra purificatrice. Avuto il filmato, Wikileaks lo ha decrittato e messo a disposizione (Fig. 2). In conclusione: "Wikileaks exists to help you safely reveal important material to the word./ We have an unbroken record in protecting confidential sources./ Contact us." Segue la firma/rinvio al sito: www.wikileaks.org.

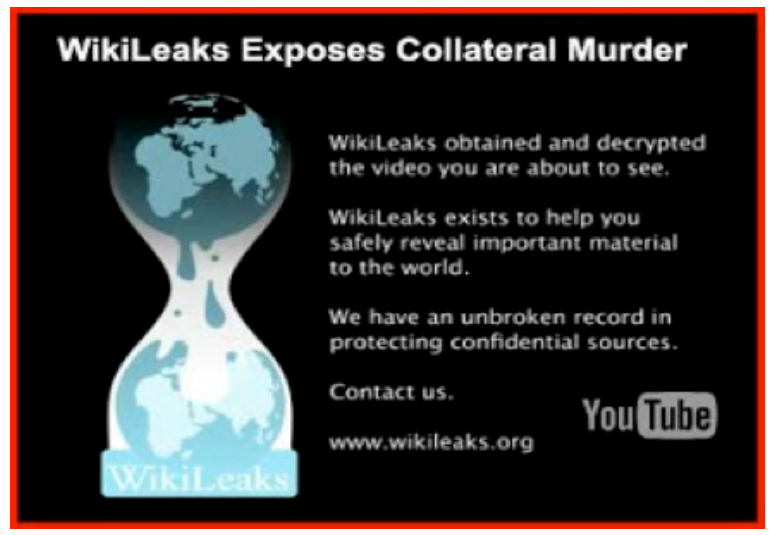

Fig.2. La rivendicazione di "autorialità" che conclude il prologo di "Collateral murder".

All'anonima e fredda visione da lontano e in B/N dall'elicottero degli "individuals", come vengono chiamati dal personale USA, dunque si antepone e contrappone la rievocazione e il ricordo di persone specifiche, con la loro storia e i loro affetti, nel contesto di una ricostruzione più ampia del comportamento colpevole e negazionista degli USA, contro il quale Wikileaks si propone invece come l'aiutante competente disponibile a ristabilire la verità agli occhi del mondo.

Per quanto riguarda I'EPILOGO, ha inizio a circa 5 minuti dalla fine, quando vengono inseriti alcuni cartelli a commento della seconda azione degli Apache registrata dal video, quella cioè in cui gli elicotteri distruggono il Van che si è fermato a prestare soccorso ai feriti dell'azione precedente, e inseguono e uccidono alcuni dei soccorritori. Il primo cartello riporta una dichiarazione resa al Washington Post dal capitano James Hall, appartenente alle truppe di terra che arrivarono sul posto otto minuti dopo l'attacco, in cui afferma che il loro mezzo si fermò per evitare di passare sopra i corpi che erano disseminati tutt'intorno. Le immagini che scorrono subito dopo questa affermazione mostrano invece che i mezzi non si fermarono, e che la cosa fu commentata ridendo dai soldati quando se ne accorsero. Un altro cartello informa della scoperta dei due bambini feriti a bordo del Van, e della decisione - sempre documentata dal sonoro del video - di portarli all'ospedale civile anziché dalla base USA, dove secondo Wikileaks avrebbero avuto cure migliori. 
Segue in un altro cartello la dichiarazione del maggiore americano Brent Cummings, che nega vi sia mai stata uccisione deliberata di civili, e afferma di non sapere come siano rimasti feriti i due bambini. Infine è riportata la testimonianza di un altro fotografo di guerra, Amhad Sahib di France Presse, rimasto in contatto con i giornalisti uccisi fino al momento della sparatoria. Secondo la sua testimonianza, gli elicotteri si misero a sparare anche a lui quando raggiunto il luogo dell'azione era sceso dalla macchina per scattare delle foto. Infine un cartello dedica il video alle famiglie delle vittime e a tutti i morti causati dalla guerra e rimasti sconosciuti. Seguono i credits, tutti in chiaro e molto espliciti salvo il primo: "Our generous source", che, poi si saprà, era Bradley Manney.

Il corpo del video viene ottenuto attraverso un'opera di SELEZIONE DELLA REGISTRAZIONE, attraverso il TAGLIO e il MONTAGGIO - percepibili alla visione - delle fasi più drammatiche della missione registrata. Inoltre vi è una TRASCRIZIONE dell'AUDIO, inserito in forma di SOTTOTITOLI in inglese (Fig. 3). La diretta ISCRIZIONE dell'ISTANZA ENUNCIAZIONALE avviene infine con la sovrimpressione sulle immagini video di nomi e segni indicali (Fig. 4) che disambiguano la visione, individuando gli attori, le azioni in corso eccetera. Va inoltre sottolineato che ogni scritta sui cartelli porta sottoscritta, in modo nascosto ma che può essere visualizzato, l'etichetta metalinguistica che la definisce come citazione, dichiarazione, oppure pensiero, interpretazione e così via.

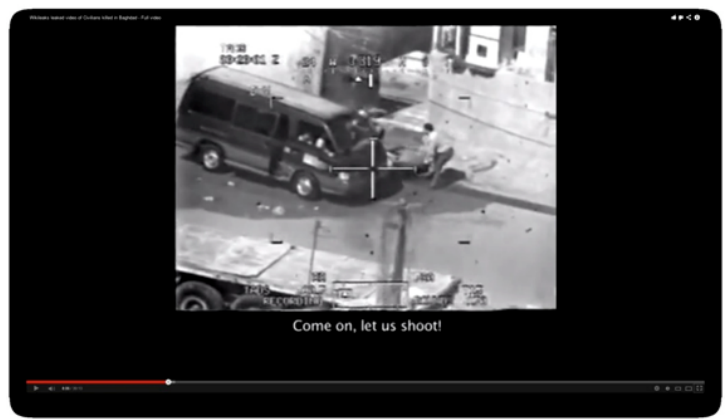

Fig.3. Un frame della riproduzione del video con l'aggiunta delle didascalie.

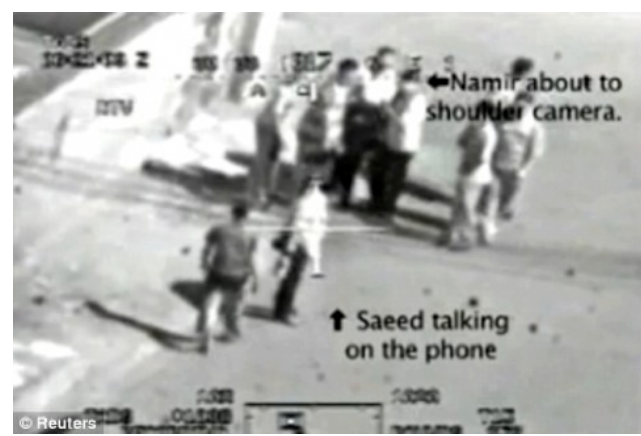

Fig.4. Un esempio degli interventi enunciazionali sul video 
Dal video emerge una precisa strutturazione narrativa dei fatti, un racconto che si può cogliere nelle fasi del suo svolgimento a partire dalla stessa prospettiva dei soldati americani che stanno sorvolando la zona e comunicando con il loro comando a terra, di grande impatto per la sua stessa qualità di "immagine rubata", di candid camera a posteriori, che svela molte cose al di là di ciò che mostra nello specifico. E cioè ad esempio il potere di controllo del territorio dei mezzi da guerra americani e l'analiticità della loro applicazione; il cinismo dei militari e il loro linguaggio letteralmente da caserma in situazioni in cui sono in gioco vite umane; la scarsissima considerazione in cui in ogni casi esse sono tenute, la mancanza di rispetto per le azioni di soccorso e per i corpi dei caduti.

Il video inoltre mette in scena un conflitto di interpretazioni: quella dei militari USA durante l'azione, secondo la quale si arriva in fretta alla conclusione che tutti gli individui coinvolti sono nemici, smentita dalla stessa sequenza dei fatti ripresi; e quella degli altri interpreti, testimoni diretti o indiretti, nella controlettura messa a punto da Wikileaks. Essi si contrappongono alle dichiarazioni USA, che dunque dalla visione del film emergono mendaci una seconda volta, come se stessero negando l'evidenza dei fatti da loro stessi documentati. La strategia di enunciazione adotta un'esplicita argomentazione intermediale: nel film le immagini video funzionano alternativamente come prova o smentita di quanto è enunciato nei testi sui cartelli di apertura.

Ed è interessante notare come si assista a un procedimento in qualche modo inverso rispetto a quello cui ci ha abituato un certo cinema, spesso di denuncia, in cui le immagini fotografiche o i materiali documentali delle situazioni ricostruite o narrate nel film vengono inserite, spesso alla fine, per produrre nello spettatore, una sorta di choc percettivo e quindi un effetto di realtà e di verità molto forte, e del tutto intermediale, come ha ben dimostrato Pietro Montani (2010).

Qui è il documento ad essere il corpo portante del film, e le inserzioni intermediali in qualche modo lo obbligano a mostrare e testimoniare, anche suo malgrado, un po' come accade con le intercettazioni telefoniche. Nel complesso il video programma una forte patemizzazione dell'enunciatario, comunque coinvolto in quello che abitualmente si definisce uno "spettacolo del dolore" (BOLTANSKI, 1997), cioè la visione impotente e a distanza dell'uccisione di persone ignare, definite come vittime, per di più in presa diretta e a partire dalla prospettiva dell'uccisore, il che può produrre sentimenti ambigui e senso di colpa, e favorire di riflesso l'indignazione e il desiderio di combattere il persecutore. Senza contare il parallelo riconoscimento di giusto informatore che ne ricava Wikileaks.

La storia di Collater murder non finisce con la conferenza stampa in cui il video fu mostrato la prima volta. Esso fu infatti caricato sul sito di Wikileaks in un apposito spazio "metalinguistico", dove permane tuttora: www.collateralmurder.com (si calcola che il video sia stato visto da oltre quattordici milioni di persone). Nel sito (Fig. 5) un testo di apertura e chiusura incornicia le due versioni del video, quella rielaborata di cui abbiamo appena parlato (short version) e quella originale (full version), a cui se ne aggiunge però 
una terza, che include lo stesso video, questa volta inframmezzato dalla testimonianza, sempre videoregistrata, di un ex-soldato USA, Ethan McCord, che ha partecipato a questo tipo di azioni e che, in divisa, commenta quella in questione. Infine, un testo conclusivo.

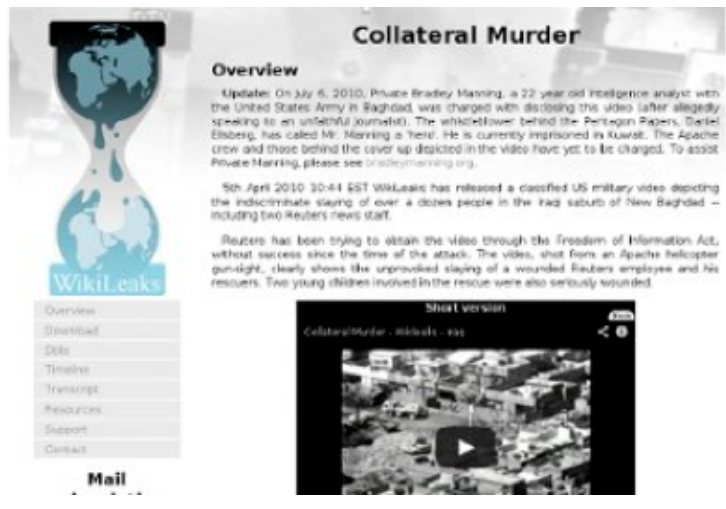

Fig.5. La pagina internet che contiene le diverse versioni del video.

I testi riprendono e in parte rielaborano quelli già visti sui cartelli all'interno del film. Sullo stesso sito si trovano molte altre cose, fra le quali una sezione "STILLS" nella quale sono riportati i fotogrammi salienti delle azioni USA di quel giorno, con una didascalia esplicativa sotto ogni immagine; un'altra sezione in cui invece viene fornito il minutaggio preciso accanto all'audio; e, ancora, le traduzioni della "conversazione" tra i militari USA prima e durante l'azione in varie lingue. Quest'ultime sono offerte da volontari che aderiscono all'invito di Wikileaks di contribuire allo scopo enunciato del suo attivismo, "quello della trasparenza da parte dei governi quale garanzia di giustizia, di etica e di una più forte democrazia". Ci sono infine molte possibilità, legate ai social media più diffusi, di condividere, diffondere e commentare i materiali del sito, e infine di inviare le proprie donazini per sostenere l'operato di Wikileaks.

Il testo verbale che nel sito incornicia i tre video ovviamente presenta Wikileaks come il Destinatore di questa "operazione trasparenza". Al di là dello stile apparentemente informativo, si tratta in realtà di un testo molto argomentato, inteso a dimostrare fra l'altro la competenza comunicativa di Wikileaks. Wikileaks è presentato ora come il soggetto, I'Agente performatore di tutta I'operazione. Questo implica un radicale cambiamento di posizione attanziale rispetto alla prima fase della sua storia, in cui come abbiamo visto si presentava soprattutto come un "Aiutante competente", e si proponeva come tale ai possibili whistleblower esterni, come nello slogan dallo stile molto pubblicitario: "Wikileaks/global defence of source and press freedom, circa now. Have documents the world needs to see? We help you safely get the truth out." (Fig. 6) 


\section{WikiLeaks \\ Manday 5 October, 2060 \\ Haws documents the world needs to seef \\ We help you safely get the truth out.}

Fig.6. Una "pubblicità" di Wikileaks.

Il testo verbale del sito Collateral murder ne ricapitola la vicenda e soprattutto sanziona positivamente l'operato di Wikileaks in tutti i diversi passaggi, ivi compresa la elaborazione del video, che in effetti è qui immediatamente possibile confrontare/ controllare con la registrazione originale. Ecco che la trasparenza comunicativa tante volte evocata si materializza nell'esplicitazione coerente delle proprie mosse nella guerra dell'informazione, nella assunzione delle relative responsabilità e nell'orgogliosa affermazione di avere adottato una strategia vincente.

Il testo inizia in realtà con un aggiornamento (del 6 luglio 2010) sulle conseguenze toccate a Bradley Manney per aver consegnato il video a Wikileaks. Egli è contrapposto come "eroe" - anche a detta di un testimonial importante come Daniel Ellsberg - ${ }^{4}$ ai soldati americani mai indagati pur avendo commesso i fatti denunciati: la denuncia è dunque perseguita dagli USA più del fatto denunciato.

La pubblicazione dei video è presentata non solo come mossa vincente nella guerra di informazione con l'esercito degli Stati Uniti, ma è utilizzata anche come prova interna al proprio discorso: si enuncia ciò che vi si vede - e che lo spettatore vedrà, se sta per la prima volta consultando il sito.

Si propone inoltre una sorta di pagina pubblicitaria dell'etica professionale di Wikileaks: il video è anche la prova dell'attenzione che quest'ultimo riserva al materiale che riceve, del suo scrupolo nell'asseverarne la provenienza e la veridicità:

Wikileaks ha ottenuto questo video, nonché i documenti giustificativi da un certo numero di militari informatori. Wikileaks fa di tutto per verificare l'autenticità delle informazioni che riceve. Abbiamo analizzato le informazioni su questo incidente da una varietà di fonti. Abbiamo parlato con testimoni e giornalisti direttamente coinvolti nell'incidente.

4 Importante economista americano liberal, fu protagonista di un importante caso di "svelamento" dei segreti dell'amministrazione americana riguardo la strategia della guerra in Vietnam. Infatti nel 1969 fotocopiò e in seguito consegnò alla stampa i cosiddetti Pentagon Papers. Si è espresso a favore di Wikileaks e di Assange. 
Questo caso riguarda nello specifico anche l'uccisione di giornalisti, cioè di coloro che abitualmente svolgono professionalmente questi compiti a beneficio del pubblico. Il testo ha qui un duplice obiettivo: denunciare il trattamento riservato durante la guerra dai militari USA ai giornalisti, e rivolgere una forma di captatio benevolentia alla categoria di questi ultimi, con la quale Wikileaks in questo momento è esplicitamente alleato.

Il terzo video con le interpolazioni del testimone, mostra l'ulteriore preoccupazione di provare che quanto si afferma è vero anche attraverso il ricorso alla testimonianza diretta, di una parola mostrata nel contesto della sua enunciazione e cioè da chi la pronuncia, nel momento e nel luogo in cui è pronunciata, e in riferimento analitico a quanto si vede nel video.

Ci si può chiedere il motivo di questa ulteriore forma di veridizione, di qualcosa che apparirebbe già così ampiamente attestato attraverso i due video e la discussione pubblica di cui sono stati oggetto.

Il fatto è che per Wikileaks, non si tratta solo di ottenere la condivisione della propria interpretazione/denuncia, bensì di istituirla ulteriormente come prova a carico degli USA, costituendosi al tempo stesso come "archivio indipendente". La raccolta e la messa in pubblico della testimonianza del soldato rientra perfettamente in questa strategia, che fra I'altro permette di "mettere una faccia" nel dialogo istituito con "il popolo della rete", cioè di sostenere e se possibile sostituire l'impersonalità di documenti che comunque come si è visto "vanno fatti parlare" con una persona che narra "un racconto autobiograficamente documentato di un avvenimento passato". Così Paul Ricoeur (2003) caratterizza l'atto del testimoniare: il testimone si autodesigna come tale e al tempo stesso asserisce la realtà di ciò su cui testimonia. Dicendo "io c'ero" si pone come terzo rispetto a tutti gli altri attori della vicenda, instaura una dimensione dialogica e fiduciaria all'interno della quale chiede di essere creduto, si pone in uno spazio pubblico che, eventualmente, può divenire luogo di controversia. Il testimone, continua ancora Ricoeur, deve essere disposto a reiterare la sua testimonianza: e quale migliore possibilità di reiterazione potrebbe esserci rispetto quella di riavviare un video da un sito internet, spazio pubblico per eccellenza?

Siamo in presenza, in effetti, di un'abile strategia veridittiva del tutto interna al testo, ma non per questo meno significativa o efficace.

Wikileaks perfeziona così la sua trasformazione attanziale: da Soggetto diventa ora Destinante sanzionatore, il custode dei valori per la salvaguardia dei quali, inizialmente, si poneva come un semplice Aiutante. Ed è interessante notare come questa trasformazione discorsiva vada di pari passo con quella, negativa e corrispettiva del proprio Antisoggetto, che viceversa perde il suo statuto iniziale e si rivela nel ruolo di Antidestinante, in base alla struttura polemica del racconto.

Isabella Pezzini è professore di Filosofia e Teoria dei Linguaggi e insegna Semiotica presso il Dipartimento di Comunicazione e Ricerca sociale della Sapienza 
Università di Roma. Fa parte del collegio docente del dottorato di Scienze della Comunicazione della stessa universit? e del Comitato scientifico del Dottorato di semiotica di Bologna. Ha conseguito il dottorato a Bologna sotto la direzione di Umberto Eco e ha studiato a Parigi con Algirdas Julien Greimas. E' responsabile di un progetto di ricerca internazionale sulla scrittura e riscrittura urbana. Fra i suoi interessi vi sono l'estensione della teoria della narrativit? e del discorso in campo sociosemiotico e di semiotica della cultura. I suoi ultimi libri sono: Introduzione a Roland Barthes (2014); Semiotica dei nuovi musei (2011) e Immagini quotidiane. Sociosemiotica visuale (2008).

isabella.pezzini@uniroma1.it www.isabellapezzini.it

\section{Referências}

BOLTANSKI, L., La souffrance à distance, Paris, Métailié, 1993.

GENETTE, G. Seuils. Paris: 1987.

LOZANO, J. El secreto. Revista de Occidente, n. 374-375, julio-agosto, 2012.

MARRONE, G. Introduzione alla semiotica del testo. Bari-Roma: Laterza, 2011.

MONTANI, P. L'immaginazione intermediale: perlustrare, rifigurare, testimoniare il mondo visibile, Bari-Roma: Laterza, 2010.

RICOEUR, P. La memoire, l'histoire, l'oubli, Paris: Seuil, 2000.

Recebido e aprovado em outubro de 2014.

\section{Anexo}

Il testo inglese del sito COLLATERAL MURDER

\section{Overview}

Update: On July 6, 2010, Private Bradley Manning, a 22 year old intelligence analyst with the United States Army in Baghdad, was charged with disclosing this video (after allegedly speaking to an unfaithful journalist). The whistleblower behind the Pentagon 
Papers, Daniel Ellsberg, has called Mr. Manning a «hero`. He is currently imprisoned in Kuwait. The Apache crew and those behind the cover up depicted in the video have yet to be charged. To assist Private Manning, please see bradleymanning.org.

5th April 2010 10:44 EST WikiLeaks has released a classified US military video depicting the indiscriminate slaying of over a dozen people in the Iraqi suburb of New Baghdad -- including two Reuters news staff.

Reuters has been trying to obtain the video through the Freedom of Information Act, without success since the time of the attack. The video, shot from an Apache helicopter gun-sight, clearly shows the unprovoked slaying of a wounded Reuters employee and his rescuers. Two young children involved in the rescue were also seriously wounded.

\section{WikiLeaks' Collateral Murder: U.S. Soldier Ethan McCord's Eyewitness Story}

The military did not reveal how the Reuters staff were killed, and stated that they did not know how the children were injured.

After demands by Reuters, the incident was investigated and the U.S. military concluded that the actions of the soldiers were in accordance with the law of armed conflict and its own "Rules of Engagement".

Consequently, WikiLeaks has released the classified Rules of Engagement for 2006, 2007 and 2008, revealing these rules before, during, and after the killings.

WikiLeaks has released both the original 38 minutes video and a shorter version with an initial analysis. Subtitles have been added to both versions from the radio transmissions.

WikiLeaks obtained this video as well as supporting documents from a number of military whistleblowers. WikiLeaks goes to great lengths to verify the authenticity of the information it receives. We have analyzed the information about this incident from a variety of source material. We have spoken to witnesses and journalists directly involved in the incident.

WikiLeaks wants to ensure that all the leaked information it receives gets the attention it deserves. In this particular case, some of the people killed were journalists that were simply doing their jobs: putting their lives at risk in order to report on war. Iraq is a very dangerous place for journalists: from 2003- 2009, 139 journalists were killed while doing their work. 